\title{
Special Issue: 15th amine oxidase conference: re-examine the amines
}

\author{
Christian Carpéné • Angelo Parini
}

Published online: 7 April 2013

(C) Springer-Verlag Wien 2013

From the 16 to 18 July 2012, more than 80 scientists from 20 different countries participated to the international Amine Oxidase Conference held in Toulouse, France.

This 15th edition of a biennial workshop organized since 1984 was an invaluable opportunity for discussing about novel insights relative to enzymes that were initially characterized to regulate neurotransmitter levels, at least those belonging to the family of biogenic amines. Thereafter, the monoamine oxidases (MAO) and other amine oxidases (AOs) have been recognized to belong to an even larger family, to work with different co-factors, to act on a wider variety of substrates, and to participate in an increasing number of physiological functions. The AOC2012 was held at the "Hôtel de Region Midi Pyrénées" and a session was shared with the 4th Food and Health symposium, since, in an attempt to re-examine the amines, several presentations have introduced unexpected substrates, such as histone $\mathrm{H} 3$, that is deaminated on its lysine 4 by Lysyl oxidase-like 2 (LOXL2) or the intestinal histamine brought by ingestion of food items with questionable freshness or poor microbial quality, the removal of which can be performed by addition of engineered vegetal or bacterial diamine oxidase (DAO). Happily, there was no need to use such nutritional supplements with the "mediterranean food" provided by the restaurants selected for social programme. The wine and cheese tasting was surely a strong source of dietary amines, but was also an opportunity to learn that in French, AOC means "Appelation d'Origine Controlée" a label of food quality. However and unfortunately, this 15 th edition missed several of the

C. Carpéné $(\varangle) \cdot$ A. Parini

INSERM U1048, Institut de Médecine Moléculaire de Rangueil (I2MC), Université Paul Sabatier, Toulouse, France

e-mail: christian.carpene@inserm.fr pioneers who have stimulated the interest in chemistry and pharmacology of amine oxidation and disseminated their importance to Life Sciences. Several of them are still active, studying various aspects of these fascinating AOs, trying to solve the complex nomenclature of the growing AO family, or deciphering unexpected properties of their "novel" substrates or inhibitors. So, let us pass in a few words all our admiration. Thus, thanks to Jean Shih, Keith Tipton, Moussa Youdim, Peter Yu, Frans Boomsma and several others who could not attend personally to this conference. More sadly, we also think in late Franca Buffoni who passed in September leaving us as legacy her achievements in copper-containing amine oxidase research. We will be influenced for a long while by her human qualities and by the future perspectives she has introduced for the functions and pharmacology of semicarbazidesensitive amine oxidase (SSAO), previously named BzAO, and presently called PrAO or VAP-1.

But, accordingly to the adage, the show has gone on, and young investigators have presented in Toulouse recent findings in an attractive manner, several of them being awarded by poster prizes. "Less younger" researchers also focused interest on AOs, by describing to the international audience, several novel functions of amine metabolism in inflammation, neurotransmission, cognition, metabolism, differentiation, digestion, endocrinology, reproduction, and cancer. From the cumulative demonstration of the importance of VAP-1 inhibition for novel anti-inflammatory therapy to the bridge between inflammation and carcinogenesis that is supported by polyamine oxidation mediated by spermine oxidase (SMO), the connections between endogenous and dietary amines and their oxidases were multiplied during the conference. The importance of lysine-specific demethylases (LSDs) or unexpected consequences of the invalidation of MAOs orientates the current 
interest in amine oxidase research to a promising contribution to cancer and ageing treatments.

This Special Issue of the Journal of Neural Transmission contains twenty-three contributions submitted by participants at AOC2012. The reader's attention will be focused first in decoding the interactions of inhibitors or substrates with monoamine oxidases, then in following the expanding functions of the above-mentioned diverse amine oxidases. In the first part, novel in silico approaches of the docking of molecules nearby the active site will be reported together with the description of novel pyrazoline derivatives as MAO inhibitors, followed by the novel insights into the use of multitarget molecules developed for neuroprotection. Original data about peripheral MAOs will close this section, indicating that, among the aldehydes generated during amine oxidation, several might exert a messenger function greater than expected. Then, in the second part, multiple aspects of the links between SSAO/ VAP-1 and inflammation will be developed, aiming at evidencing the benefits of SSAO/VAP-1 inhibitors as antiinflammatory agents. Descriptive works on lysyl oxidase and diamine oxidase will precede a short focus on an old molecule, phenelzine, which reveals unusual actions. Last, reports on novel tools useful for studying the regulation, activity, or abundance of several copper-containing amine oxidases will close this multifaceted selection.

It is hoped that the exciting contributions compiled in the following pages of this volume will encourage an increasing number of readers to attend to the next Amine Oxidase Workshop, in 2014, which will be hosted by Wolfgang Jarolimek and Ian Mac Donald in Sidney, Australia.

We are grateful to Dr. Peter Riederer, Editor of the Journal of Neural Transmission, for his invaluable help making this Special Issue possible. Finally, thanks to Andrew Holt, organiser of the previous meeting, to all the members of scientific advisory board, and to our sponsors. 